\title{
Nonsinonasal Neuroendocrine Carcinoma of the Head and Neck: Case Series and Review of Literature
}

\author{
${ }^{1}$ Duncan F Hanby, ${ }^{1}$ Andrew McWhorter, ${ }^{1}$ Eric Wallace, ${ }^{2}$ Eugene A Woltering, ${ }^{3}$ Anthony Harton, ${ }^{4}$ Kerri \\ B Santiago, ${ }^{1}$ Daniel W Nuss, ${ }^{1}$ Rohan R Walvekar \\ ${ }^{1}$ Department of Otolaryngology and Head-Neck Surgery, LSU Health Sciences Center, New Orleans, LA, USA \\ ${ }^{2}$ Department of Surgery, Section of Endocrine Surgery, LSU Health Sciences Center, New Orleans, LA, USA \\ ${ }^{3}$ Department of Pathology, (Pathology Group of Louisiana) Our Lady of the Lake Regional Medical Center, Baton Rouge, LA, USA \\ ${ }^{4}$ Department of Pathology, University Medical Center, Lafayette, LA, USA
}

Correspondence: Rohan R Walvekar, Assistant Professor, Department of Otolaryngology and Head-Neck Surgery, LSU Health Sciences Center, 533 Bolivar Street, Suite 566, New Orleans, LA 70112, USA, Phone: (504) 568 4785, Fax: (504) 568 4460 e-mail: rwalve@lsuhsc.edu

\begin{abstract}
Objective: To describe three patients with nonsinonasal neuroendocrine carcinoma (NSNEC) of the head and neck and present a systematic review of literature.

Study design: Retrospective chart review

Methods: Three patients with pathologically proven NSNEC of the head-neck who presented to our institution were identified. Relevant demographic, clinic-pathological, and radiological data was recorded. A web-based search was conducted to identify relevant scientific literature on "neuroendocrine carcinoma (NEC) of the head and neck" and a systematic review of literature is presented.

Results: Two female and one male patient aged 44 to 66 years presented to our service with NEC of the supraglottis (2/3), and of the thymus (1/3). Diagnosis was confirmed with immunohistochemical staining such as Ki-67, synaptophysin, chromogranin, and also with octreotide scanning. The first case, a T4bNOMO large cell NEC of the thymus with tracheal invasion received conservative airway management and emergent radiotherapy. The second case, moderately differentiated NEC of the supraglottis was managed with a laser supraglottic laryngectomy followed by adjuvant treatment. The third case, a laryngeal NEC was treated with chemoradiotherapy.

Conclusions: NSNEC of the head and neck are extremely rare tumors with variability in clinical presentation that present challenges in diagnosis and treatment planning. Consideration of the histological subtype and staining characteristics of the individual tumor, accurate diagnosis, and classification of the tumor is vital in order to tailor therapeutic intervention.
\end{abstract}

Keywords: Neuroendocrine carcinoma, head-neck, nonsinonasal, review of literature.

\section{INTRODUCTION}

Nonsinonasal neuroendocrine carcinomas (NSNECs) of the head and neck are extremely rare. Within the head and neck, neuroendocrine carcinomas (NECs) as a group are found most commonly in the larynx comprising 0.5 to $1.0 \%$ of all laryngeal neoplasms and occur second in frequency only to squamous cell carcinoma, the most predominant pathology within the larynx. Histologic subtypes within this genre of malignancy range from the favorable but rare welldifferentiated carcinoid to more aggressive and widely metastatic small cell carcinoma. ${ }^{1,2}$ These unique and rare neoplasms present a variety of diagnostic and treatment dilemmas to the head and neck surgeon. We present three cases of NSNEC of the head-neck with a relevant systematic review of literature.

\section{METHODS}

A retrospective chart review was conducted of three consecutive patients diagnosed with NSNEC of the head and neck. The study was conducted at the Department of Otolaryngology Head and Neck Surgery, Louisiana State University Health Sciences Center, New Orleans, LA. Relevant demographic, clinic-pathological, and radiological data was recorded. A web-based search was conducted to identify relevant scientific literature on "neuroendocrine carcinoma (NEC) of the head and neck" in order to present a systematic review of current literature. Keywords that were entered included, "neuroendocrine carcinoma, head and neck, nonsinonasal neuroendocrine carcinoma, NSNEC, and NEC". 


\section{CASE SERIES}

\section{Patient 1}

The first patient is a 66-year-old female who initially presented to the emergency room with biphasic stridor, expiratory greater than inspiratory. The head and neck examination including a flexible laryngoscopy was unremarkable. However, a computerized tomography (CT) scan of the neck demonstrated a large, poorly circumscribed mass which initially appeared to be emanating from the right lobe of her thyroid gland. The mass extended into her superior mediastinum with tracheal invasion $4 \mathrm{~cm}$ superior to the carina. A diagnostic ultrasound guided true-cut needle biopsy revealed a high grade NEC. Shortly after her true cut biopsy, she had an abrupt change in her voice and was noted to have right true vocal fold motion impairment. Immunohistochemical stains were positive for cytokeratin, CD56, chromogranin and minimal staining with synaptophysin (Figs 1A to C). The lesion did not stain positive for calcitonin. She was staged as a T4N0M0 large cell neuroendocrine carcinoma based on clinical evaluation and imaging studies. In view of a tumor that was assessed to be technically unresectable, the multidisciplinary tumor conference recommendation was made to offer treatment with chemotherapy and radiation. At her six month followup, her stridor has resolved and she has regained the motion of her right true vocal fold.

\section{Patient 2}

A 49-year-old African American female who presented to our service in respiratory distress was found to have a supraglottic mass and right-sided cervical lymphadenopathy. A diagnostic pan-endoscopy and biopsy confirmed our clinical findings and pathology revealed a well-differentiated carcinoid tumor. The patient underwent a transoral laser partial laryngectomy. Final histopathology was consistent with a moderately differentiated (atypical) neuroendocrine carcinoma with positive margins at the thyroid cartilage, as well as lymphovascular and neural invasion. Immunohistochemical staining showed immunoreactivity for Ki-67, (8-10\%), Synaptophysin (95-99\%), and Chromogranin $(50 \%)$, (Figs $2 \mathrm{~A}$ to $\mathrm{C})$. An Octreotide scan was also
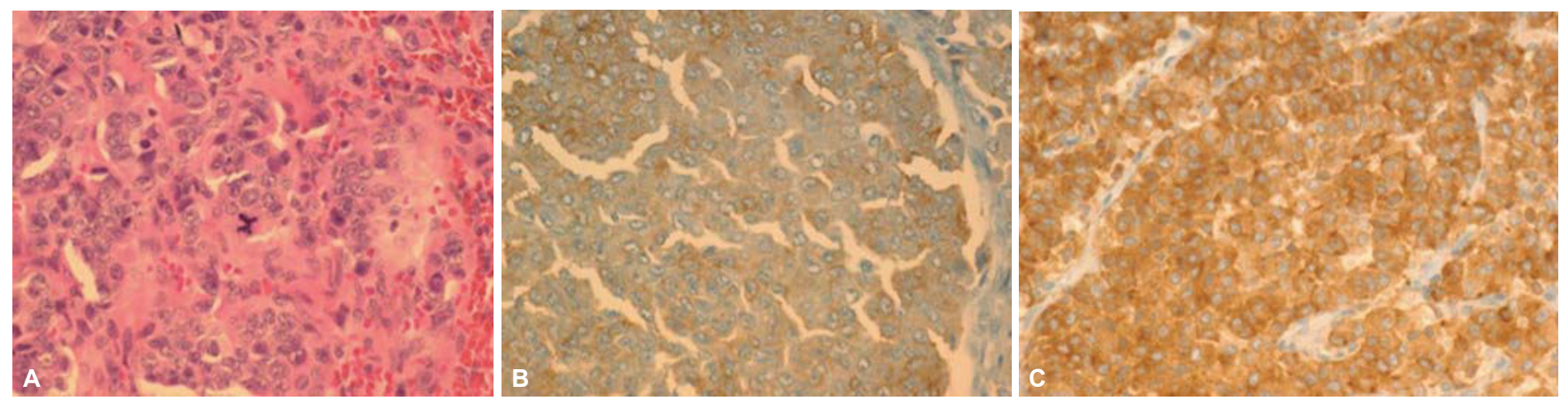

Figs 1A to C: (A) Hematoxylin and Eosin Stain (400x) demonstrating the tumor in patient 2 composed of nests and cords of neuroendocrine cells with fairly abundant eosinophilic cytoplasm. The nuclei had a neuroendocrine appearance with fairly evenly dispersed chromatin. However, a significant population of cells showed cellular atypia in the form of nuclear pleomorphism and prominent nucleoli. Increased mitotic activity was indentified as were atypical mitotic figures. The tumor cells were positive for cytokeratin cocktail and neuroendocrine markers synaptophysin (B), and chromogranin $(\mathrm{C})$
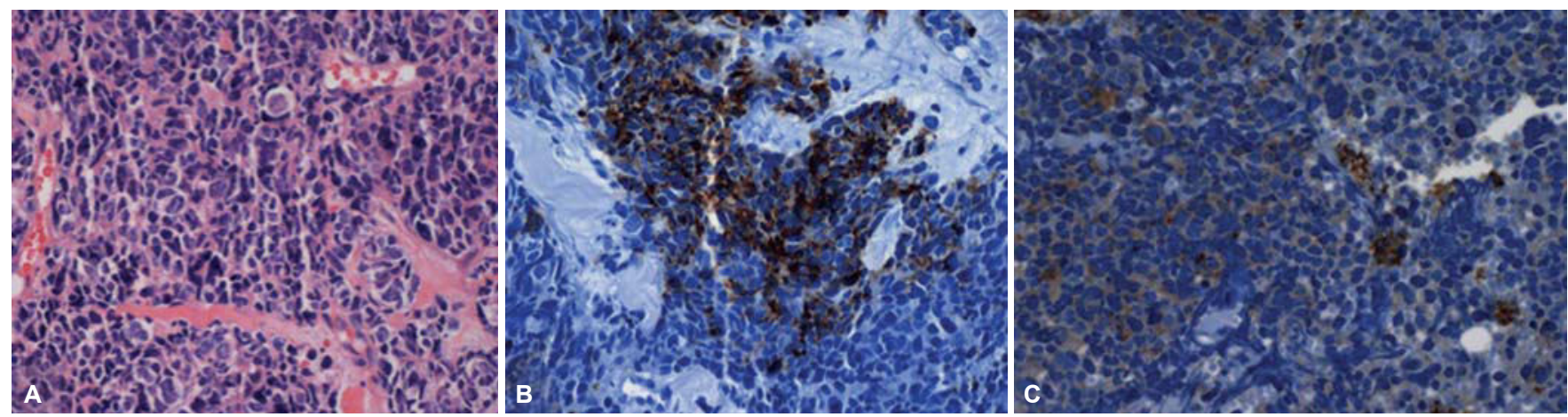

Figs 2A to C: (A) Hematoxylin and Eosin Stain (400x) demonstrating the tumor in patient 1 composed of large cells, with prominent oval to pleomorphic nuclei and speckled to clumped chromatin. Numerous mitoses are identified. Necrosis is present. Immunohistochemical stains are positive for cytokeratin, CD56, chromogranin (B) and minimal staining with synaptophysin (C). The histologic and immunohistochemical stains are most consistent with a high grade neuroendocrine carcinoma 
performed which demonstrated a $3.7 \mathrm{~cm}$ right sided neck mass of somatostatin rich tissue consistent with a lymphatic metastasis from her primary tumor. The multidisciplinary tumor conference recommendation was upfront surgical therapy that amounted to a total laryngectomy and bilateral neck dissections. The patient declined having the laryngectomy and consequently underwent bilateral selective neck dissections followed by chemoradiation. The patient followed up on a monthly basis in the postoperative period and remains free of disease at her last follow-up.

\section{Patient 3}

The third patient was a 44-year-old male who presented in November 2007 with a seven month history of progressive hoarseness. In addition, the patient reported new onset development of several tender erythematous lesions distributed throughout his scalp and a left supraorbital lesion. Examination revealed multiple $0.5 \mathrm{~cm}$ skin nodules distributed throughout his scalp with palpable occipital and jugular lymphadenopathy. Flexible fiberoptic laryngoscopy demonstrated a polypoid, fungating epiglottic mass extending from the petiole of the epiglottis to the left false vocal fold. Biopsy of one of the scalp lesions was nondiagnostic. The patient underwent a tracheotomy and staging pan-endoscopy with biopsy that revealed a poorly differentiated neuroendocrine carcinoma of the supraglottis. Immunohistochemical stains revealed positivity for chromogranin and synaptophysin as well as CD56 (neural cell adhesion molecule). The patient was referred for chemotherapy and radiation in view of widespread distant metastatic disease and succumbed to his disease at 16 months after initial diagnosis.

\section{DISCUSSION}

Head and neck cancers account for approximately five percent of all cancers. The vast majority of primary malignancies within the head and neck are squamous cell carcinomas. In her review of the SEER database, Davies showed that squamous cell carcinoma accounts for greater than $80 \%$ of cancers of the lip, tongue, tonsil, oropharynx, hypopharynx, larynx, and cervical esophagus, and $57 \%$ of cancers in the nose. ${ }^{3}$ NSNEC of the head and neck are exceedingly rare. The most commonly involved nonsinonasal site within the head and neck is the larynx, yet this histologic group still only comprises 0.5 to $1.0 \%$ of all laryngeal neoplasms. A retrospective review of patients treated at a major, tertiary, high volume cancer center only yielded 23 NSNEC patients over 17 years. ${ }^{1}$

The majority of these tumors fall into three histological subtypes: well-differentiated (typical) NEC (i.e. carcinoid tumors), moderately differentiated (atypical) NEC, and poorly differentiated (small cell) NEC. Well-differentiated typical carcinoid tumors are the least common with only 20 case reports currently in the literature. ${ }^{4}$ Histological subtype in these tumors is very important as each has a direct influence on clinical behavior, appropriate treatment and prognosis.

Well-differentiated NECs of the larynx are reported to have excellent overall outcomes with surgical extirpation. The incidence of nodal metastasis for well-differentiated NEC is low and consequently, there does not appear to be a role for elective neck dissection or adjuvant therapy in the treatment of these rare lesions. ${ }^{4}$

The experiences of the head and neck community are more variable with regard to treatment of more advanced NEC of the head and neck. Ferlito et al, recommend surgical therapy for atypical (moderately differentiated) NEC of the larynx with level II-IV neck dissections even in clinically node negative necks due to the because of the high incidence of regional metastasis with more advanced disease. ${ }^{4}$ There seems to be a general consensus that surgical resection is preferred for atypical carcinoid tumors in the larynx and that these tumors are fairly resistant to nonsurgical treatment options. However, in a series from MD Anderson Cancer Center, Gillenwater et al demonstrated some response to chemoradiation therapy. ${ }^{5}$ Our patient with atypical laryngeal NEC had initial transoral laser surgery with positive margins, perineural and lymphovascular invasion was treated with elective neck dissections and postoperative chemoradiotherapy. This patient remains free of disease despite the nontraditional treatment protocol used to treat the disease. Therefore, it is reasonable then to question: do traditional pathological features such as positive margins, perineural invasion, and lymphovascular invasion have the same impact on recurrence and survival rates for NSNECs as they do in other cancers wherein their role is better established? In addition, it would be interesting to study how these features would compare to known indicators of poor prognosis in NSNEC such as histological subtype. The mainstay of management of small cell NEC has been nonsurgical therapeutic options. Barker recommend induction chemotherapy followed by radiation as well as advocating 
prophylactic cranial irradiation (PCI) for patients who respond to the induction chemotherapy as patients in their study group experienced high rates of isolated intracranial metastases with 5 year rates of IC metastasis at $44 \%$. This led the authors to conclude that the central nervous system (CNS) may serve as a "sanctuary site" for NSNEC. ${ }^{1}$ Patients not responding to induction chemotherapy were treated with aggressive concomitant chemoradiation to try and subdue their local disease. Conversely, Baugh reported only a 7.7\% incidence of CNS metastases from laryngeal small cell neuroendocrine carcinomas and consequently did not recommend $\mathrm{PCI}^{2}$ Although, there is inadequate data to recommend PCI; there is a consensus in the majority of literature, that laryngeal small cell NEC are best treated with concomitant chemoradiotherapy. ${ }^{4,6}$

In a study of 11842 reported cases of carcinoid tumors, Soga discovered that the highest rate of metastases was noted in tumors of the ileocecum $(75.3 \%)$, followed by the jejunoileum (65.2\%), pancreas $(64.2 \%)$, and the larynx (61.4\%). In addition, they found that laryngeal carcinoid tumors had a $47.6 \% 5$ year survival rate with a $33.3 \%$ incidence of metastasis. Atypical carcinoid tumors are reported to have a $66.7 \%$ incidence of metastases. These tumors were found to metastasize most commonly to the lymph nodes, liver and lung. ${ }^{7}$ In their study, Woodruff and Senie report a 5 year survival rate of $48 \%$ for laryngeal atypical carcinoid tumors. Ferlito also reported a similar $(50 \%)$ survival outcome. ${ }^{4,6,8}$ Of all NEC subtypes, the prognosis for small cell NEC is considered to be the worst, with more than $90 \%$ of patients eventually developing metastatic disease and 5 year survival reported as low as 5\%. ${ }^{4,6,7,9}$ A recent retrospective review of nonsquamous cell malignancies in the larynx demonstrated that supraglottic NEC and small cell NEC had a mean survival of 44.8 and 52.1 months respectively. ${ }^{10}$ In a study group predominately composed of small cell undifferentiated NEC, Barker reported similar survival statistics with a 5 year overall survival of $33 \%$ and disease-free 5 year survival at $25 \%{ }^{1}$

\section{CONCLUSIONS}

Neuroendocrine carcinoma of the head and neck presents unique challenges both in diagnosis and treatment planning. Clearly, controversy still exists on the appropriate management of these tumor which stems from lack of robust scientific evidence that partly is a result of low incidence of the disease. The cases above highlight the heterogeneous behavior and presentation of these lesions. One must consider the histological subtype and staining characteristics of the individual tumor in order to tailor the appropriate therapy. Given the dearth of evidence regarding treatment options and prognostic indicators, case series such as ours continue to shed light on the nature and epidemiology of this rare pathology.

\section{ACKNOWLEDGMENTS}

The authors would like to thank Ms Deanna Loerwald and Ms Keisha C Cedano (Department of Otolaryngology Head and Neck Surgery, LSU Health Sciences Center, New Orleans, LA), for their help.

\section{REFERENCES}

1. Barker JL (Jr), Glisson BS, Garden AS, et al. Management of nonsinonasal neuroendocrine carcinomas of the head and neck. Cancer 1 Dec 2003;98(11):2322-28.

2. Baugh RF, Wolf GT, McClatchey KD. Small cell carcinoma of the head and neck. Head Neck Surg May-Jun 1986;8(5): 343-54.

3. Davies L, Welch HG. Epidemiology of head and neck cancer in the United States. Otolaryngol Head Neck Surg Sep 2006; 135(3):451-57.

4. Ferlito A, Devaney KO, Rinaldo A. Neuroendocrine neoplasms of the larynx: Advances in identification, understanding, and management. Oral Oncol Sep 2006;42(8):770-88.

5. Gillenwater A, Lewin J, Roberts D, El-Naggar A. Moderately differentiated neuroendocrine carcinoma (atypical carcinoid) of the larynx: A clinically aggressive tumor. Laryngoscope. Jul 2005;115(7):1191-95.

6. Ferlito A, Barnes L, Rinaldo A, Gnepp DR, Milroy CM. A review of neuroendocrine neoplasms of the larynx: Update on diagnosis and treatment. J Laryngol Otol Sep 1998;112(9): 827-34.

7. Soga J. Carcinoids and their variant endocrinomas. An analysis of 11842 reported cases. J Exp Clin Cancer Res Dec 2003; 22(4):517-30.

8. Woodruff JM, Senie RT. Atypical carcinoid tumor of the larynx. A critical review of the literature. ORL J Otorhinolaryngol Relat Spec. 1991;53(4):194-209.

9. Gnepp DR. Small cell neuroendocrine carcinoma of the larynx. A critical review of the literature. ORL J Otorhinolaryngol Relat Spec. 1991;53(4):210-19.

10. Lin HW, Bhattacharyya N. Staging and survival analysis for nonsquamous cell carcinomas of the larynx. Laryngoscope. Jun 2008;118(6):1003-13. 